\title{
A Two-Level Optimization Method for Hypersonic Periodic Cruise Trajectory
}

\author{
Hesong Li, Yi Wang $\mathbb{D}^{\text {, }}$, Yunfan Zhou, Shangcheng Xu, and Dan Su \\ College of Aerospace Science, National University of Defense Technology, Changsha 410073, China \\ Correspondence should be addressed to Yi Wang; 1262581502@qq.com
}

Received 23 March 2021; Revised 3 June 2021; Accepted 17 June 2021; Published 30 June 2021

Academic Editor: William MacKunis

Copyright (c) 2021 Hesong Li et al. This is an open access article distributed under the Creative Commons Attribution License, which permits unrestricted use, distribution, and reproduction in any medium, provided the original work is properly cited.

\begin{abstract}
Periodic cruise has the potential to improve the fuel-saving efficiency of hypersonic cruise vehicle but is difficult to optimize. In this paper, a two-level optimization method for the trajectory of periodic cruise is proposed. Due to that the periodic cruise trajectory can be divided into an acceleration phase where engine works and a glide phase where engine is off, the two-level optimization method is proposed to optimize the trajectory in each phase by the corresponding level. In the first level, Downhill Simplex Method (DSM) is employed to find an optimal angle of attack in the acceleration phase. Subsequently, the optimal trajectory in glide phase is obtained by the Pseudo-Spectral Method (PSM) in the second optimization level. Numerical results demonstrate the effectiveness of the proposed method. Finally, through comparing with steady-state cruise, it is concluded that periodic cruise makes full use of the change of atmospheric density and lift-drag ratio; thus, fuel saving is achieved.
\end{abstract}

\section{Introduction}

Hypersonic vehicle generally refers to the aircraft flying at Mach number above 5 [1], which has a series of advantages such as high flight altitude, fast speed, and strong penetration ability, and it has a far-reaching impact on the development of aerospace technology in the foreseeable future [2]. Therefore, the research on hypersonic technology has been widely concerned by researchers all over the world $[3,4]$.

As one of the important components of hypersonic flight technology, the design of flight trajectory is based on the aerodynamic characteristics of hypersonic vehicle and the complex flight environment, and it is aimed at minimum fuel consumption, longest range, or shortest flight time, with satisfying the complex path constraints including heating rate, acceleration load, dynamic pressure, and other terminal constraints [5]. Through reasonable design, the optimal flight trajectory can improve the efficiency of subsystem in the aircraft and reduce the cost of the whole flight. At the same time, it can provide guidance in the structural and material selection of the aircraft, which is conducive to the overall design of aircraft.

The whole trajectory of hypersonic cruise vehicle is generally divided into ascending stage, cruising stage, and gliding stage. Due to the difference of mission performed by hypersonic vehicle in each stage, the flight modes are also quite different. Therefore, it is necessary to establish appropriate performance indices for different stages, which makes the research face different problems [6]. The direction and magnitude of aerodynamic force in the ascending stage are closely related to the direction of thrust, and it is necessary to control the load caused by aerodynamic force [7]; in the cruising stage, it is difficult for hypersonic vehicle to maintain high maneuverability [8], while the constraints of terminal velocity and flight-path angle are very important to the gliding stage $[9,10]$. Therefore, many researches can be carried out to solve these problems. Generally speaking, the cruising stage accounts for a large proportion in the whole flight process of hypersonic cruise vehicle, and the flight range depends on the cruising stage to a great extent. Therefore, it is of great significance to study the design of trajectory in the cruising stage.

To make the range of aircraft longer, an effective method is to improve the fuel efficiency of aircraft in the cruising stage. Finding the cruise trajectory with the highest fuel efficiency has been the focus of many researchers [11]. In these researches, steady-state cruise and periodic cruise are two main cruise modes. Steady-state cruise refers to the cruise 
with constant altitude and speed [12]. Thus, it is relatively easy to optimize the trajectory of steady-state cruise because it is a two-degree-of-freedom (DOF) problem [13], while Irons et al. [14] have demonstrated that this trajectory is not optimal. The theoretically optimal trajectory is an infinite DOF curve, whose theoretical analysis is quite difficult as a two-point boundary value problem (TPBVP). Therefore, researchers tried to find a trajectory with better performance by dividing the cruise trajectory into a few phases which were uniform, and then, the concept of periodic cruise was raised. During periodic cruise, the trajectory approximates the form of a periodic function. The engine switches on and off according to a periodic law, which makes the powered propulsion and unpowered glide alternate, and the altitude, velocity, and other state variables change periodically. At the end of a cycle, the parameters of flight state are the same as that of the initial state [15].

There are many researches about the optimization of trajectory by theoretic analysis [16], but many of them are based on simplification; otherwise, it is difficult to carry out because of the complex characteristics of hypersonic flight. With the development of computational science and intelligent algorithm, many researchers have introduced optimization method to the field of aerospace $[17,18]$. A number of trajectory optimization methods were proposed, which were well reviewed by [19], and it is validated that optimization method is effective to solve trajectory optimization problems [20]. In many researches about the optimization of cruise trajectory, it is generally considered that the trajectory of steadystate cruise is a suboptimal initial guess for the optimization of trajectory in periodic cruise [21], and the performance index of them is always used for comparison to draw relevant conclusions [22]. Many researches find that a certain amount of fuel can be saved through periodic cruise compared with steady-state cruise, and the trajectory of periodic cruise is more flexible. However, due to the complex characteristics of hypersonic flight, trajectory optimization is a multiobjective, multiconstraint, strong-coupling, and highly nonlinear optimization problem [23], which increases the difficulty of obtaining the optimal solution. In the optimization of periodic cruise trajectory, angle of attack and throttle are the two control variables, which are also functions about time and need to be determined to minimize the objective. While these two variables are nonlinear and discontinuous sometimes, thus, it is not easy to deal with them at the same time in an optimization algorithm unless new constraints are added. So far, there is no effective indirect numerical method and professional software that can deal with the problem of periodic cruise trajectory design in a unified framework [15]. Therefore, it is significant to explore new method to optimize the trajectory of periodic cruise.

In order to obtain the optimization result of periodic cruise trajectory quickly and robustly, many researchers introduced the idea of hierarchical optimization to separate optimization variables and optimize them, respectively, by different algorithms. Earlier, Subbarao and Shippey [24] proposed a trajectory optimization method by combining the collocation method with the genetic algorithm. The initial values of variables were selected by genetic algorithm to improve the optimization efficiency, and then, the trajectory was optimized by the collocation method. Kang et al. [25] studied the optimal periodic cruise trajectory by combining genetic algorithm and direct shooting method; then, a method of two-level optimization was developed to deal with the parameters of initial state in the outer loop and the control variables in the inner loop, respectively. In recent years, many new effective two-level trajectory optimization methods have been proposed and applied in trajectory optimization. Chai et al. [26] formulated and solved a constrained space maneuver vehicle trajectory optimization problem using a three-layer-hybrid optimal control solver, and good performance was obtained. Liu et al. [6] developed a two-level optimization algorithm to solve the optimal steady-state cruise trajectory by combining PSO algorithm with sequential quadratic programming. In [27], deep neural network was trained by generated trajectories from fuzzy multiobjective transcription method; then, a two-step strategy for real-time trajectory planning was proposed with feasibility and reliability confirmed. In this paper, due to the complexity of periodic cruise trajectory design, the concept of hierarchical optimization is also employed.

Considering that there are an acceleration phase and a glide phase in a period of periodic cruise, which can provide convenience to optimize the trajectory if the two phases can be optimized separately, a two-level optimization method which deals with the optimization problems in different phases by different levels, respectively, is proposed in this paper. The first level optimizes the acceleration phase, and the glide phase is optimized by the second level, and it is required that the fuel consumption averaged by range is lowest in the period. The second part of this paper introduces the models including parameterized aircraft model and dynamic equations. The description of optimization problem as well as method is also illustrated in detail. The solution of steady-state cruise and optimized result of periodic cruise are displayed in the third part. Finally, the difference of trajectory between steady-state cruise and periodic cruise is discussed, and the fuel-saving mechanism of periodic cruise is explored by contrast with steady-state cruise.

\section{Models and Methods}

2.1. Model of Hypersonic Vehicle. The HL-20 aircraft model [28] is widely employed in the research of trajectory optimization [29], and the aerodynamic coefficients are given in (1) [30]. Mach number is denoted by $M$. The coefficient of lift and drag are denoted by $C_{L}$ and $C_{D}$, respectively. $C_{D 0}$ means the drag coefficient at zero attack of angle whose value is 0.008 when $M>10$.

$$
\left\{\begin{array}{l}
C_{L}(M, \alpha)=C_{L 0}(M)+C_{L \alpha}(M) \alpha, \\
C_{D}(M, \alpha)=C_{D 0}(M)+K(M) C_{L}^{2}, \\
C_{L 0}(M)=\frac{1}{20 \pi} \arctan [10(M-1)]-0.035, \\
C_{L \alpha}(M)=0.057 \exp (-0.654 M)+0.014, \\
K(M)=1.85[1-\exp (-0.2356 M)] .
\end{array}\right.
$$


Thrust coefficient is calculated in (2) [30], and the value of thrust force is calculated by (3), where $s$ denotes the throttle, $q$ means the aerodynamic pressure, and $S_{e}$ is the reference area of engine whose value is $9.02 \mathrm{~m}^{2}$. Angle of attack is denoted by $\alpha$, and the unit is degree.

$C_{T \max }=\left\{\begin{array}{l}0.4736 M^{1.5}+1.6947 M^{-2}(M<4), \\ \frac{15(\alpha+5)^{0.25}}{M^{1.15}} \cdot \exp \left[-\frac{M^{0.08}}{200} \times\left(\alpha+5-\frac{35}{M^{0.6}}\right)^{2}\right](M \geq 4),\end{array}\right.$

$$
T=s q C_{T \max } S_{e}
$$

The specific impulse of engine is computed by (4), and fuel consumption per second is calculated by (5), where $h$ denotes flight altitude and $g$ denotes the acceleration of gravity.

$$
\begin{gathered}
I_{\mathrm{sp}}= \begin{cases}4500-10(h-20) & (M<4), \\
-245 M+5480-10(h-20) & (M \geq 4),\end{cases} \\
\frac{d m}{d t}=-\frac{T}{g I_{\mathrm{sp}}} .
\end{gathered}
$$

The 1976 U.S. Standard Atmosphere Model is used. An altitude factor is defined by [21], where $R_{e}$ means the radius of earth.

$$
H=\frac{h}{1+h / R_{e}} .
$$

When flight altitude is in the range of 32 to $47 \mathrm{~km}$, the atmospheric density, denoted by $\rho$, is calculated in (7), where $\rho_{0}=1.225 \mathrm{~kg} / \mathrm{m}^{3}$.

$$
\begin{gathered}
W=1+\frac{H-39.7499}{89.4107}, \\
\rho=3.2618 \times 10^{-3} \rho_{0} W^{-13.2011} .
\end{gathered}
$$

For the sake of simplicity, the earth is considered to be a homogeneous sphere, and the acceleration of gravity is a constant at $9.8 \mathrm{~m} / \mathrm{s}^{2}$. The velocity of sound, which is denoted by $a$, can be regarded as a constant whose value is $340.294 \mathrm{~m} / \mathrm{s}$ [30]. Then, the dynamic model is described in (8). The flight-path angle is denoted by $\gamma . T, L$, and $D$ are thrust, lift, and drag, respectively; $m$ means the mass of aircraft whose value is $89930 \mathrm{~kg}$, and $r$ denotes the flight range. Based on (8), the trajectory of aircraft can be simulated.
TABLE 1: The limits of control variables.

\begin{tabular}{lcc}
\hline Variable & Lower limit & Upper limit \\
\hline$\alpha\left({ }^{\circ}\right)$ & 5 & 20 \\
$s$ & 0 & 1 \\
\hline
\end{tabular}

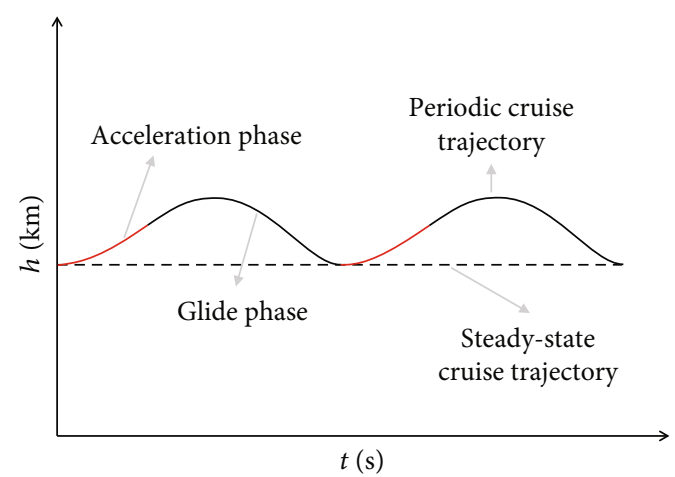

FIGURE 1: The flight plan of periodic cruise.

$$
\left\{\begin{array}{l}
\frac{d h}{d t}=M \cdot a \cdot \sin \gamma, \\
\frac{d M}{d t}=\frac{T \cos \alpha-D-m g \sin \gamma}{m \cdot a}, \\
\frac{d \gamma}{d t}=\frac{T \sin \alpha+L}{m M \cdot a}+\cos \gamma\left(\frac{M \cdot a}{R_{e}+h}-\frac{g}{M \cdot a}\right), \\
\frac{d r}{d t}=M \cdot a \cdot \cos \gamma\left(\frac{R_{e}}{R_{e}+h}\right) .
\end{array}\right.
$$

The optimization of aircraft trajectory is a kind of classic optimal control problem. For a periodic dynamic system whose period is $t_{c}$, the dynamic equation can be formulated as (9), where $x$ denotes the array of state variables and $u$ means the array of state variables.

$$
\dot{x}=f(x(t), u(t)) .
$$

The function of $u(t)$ needs to be determined in order to minimize the index function in (10) with the terminal constraints in (11) satisfied.

$$
\begin{gathered}
J=\int_{0}^{t_{c}} g(x(t), u(t)) d t, \\
x(0)=x\left(t_{c}\right) .
\end{gathered}
$$

Therefore, in the optimization of trajectory, $h, M$, and $\gamma$ are regarded as state variables, which is formulated as $x=(h, M, \gamma)^{T} . \alpha$ and $s$ are regarded as control variables, which is formulated as $u=(\alpha, s)^{T}$. And the optimization of trajectory is to determine the function of $\alpha$ and $s$ to minimize the objective which is relative to the parameters of trajectory. 


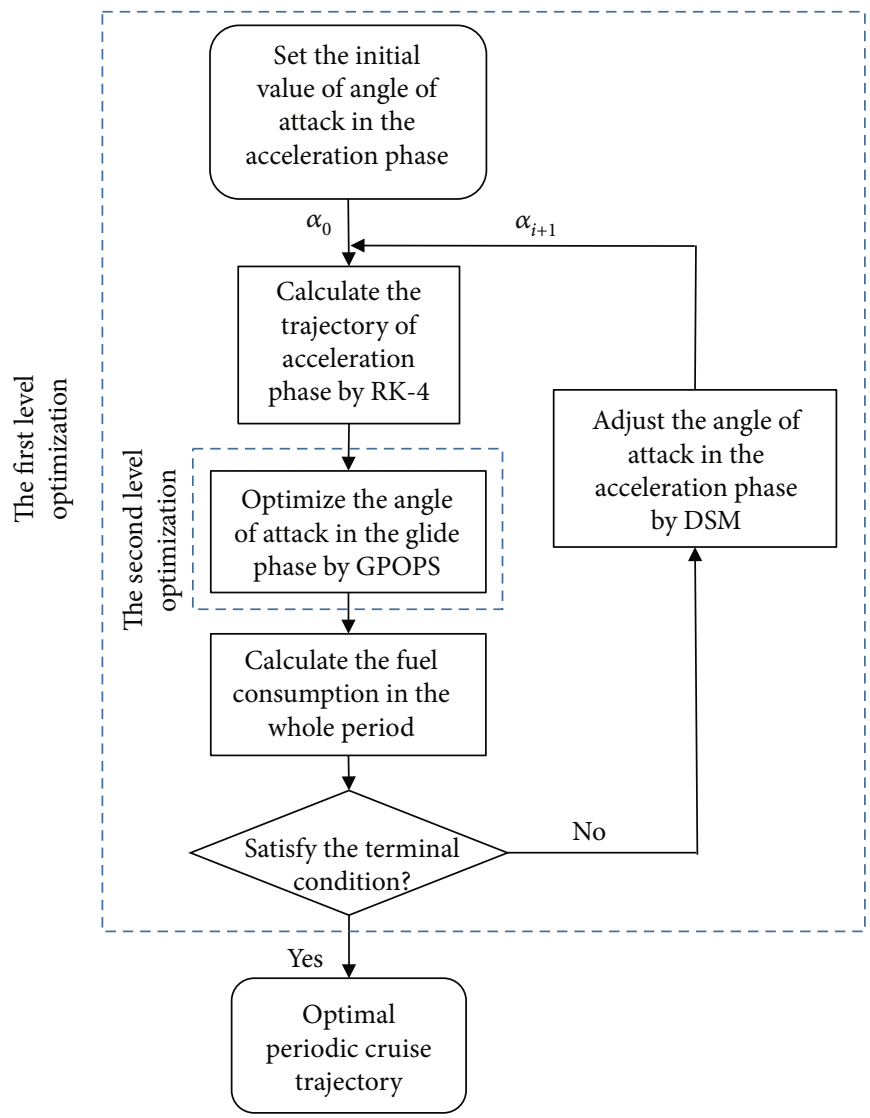

FIGURE 2: The process of two-level optimization.

2.2. Method for Steady-State Cruise. To compare with the result of periodic cruise, the trajectory of steady-state cruise needs to be solved first.

In steady-state cruise, $\gamma$ is 0 and $h$ and $M$ remain unchanged. The first three equations in (8) should be equal to 0 . While $\gamma$ is 0 so that the first formula is naturally satisfied, thus, (12) is obtained. $L$ and $D$ are related to $h, M$, and $\alpha$, while $T$ is related to $h, M, \alpha$, and $s$. Overall, there are two equations with four variables.

$$
\left\{\begin{array}{l}
\frac{d M}{d t}=\frac{T \cos \alpha-D}{m \cdot a}=0 \\
\frac{d \gamma}{d t}=\frac{T \sin \alpha+L}{m M \cdot a}+\frac{M \cdot a}{R_{e}+h}-\frac{g}{M \cdot a}=0
\end{array}\right.
$$

Given the value of $h$ and $M$, the other two variables can be solved and the fuel consumption averaged by range can also be obtained. Firstly, $T$ can be eliminated by (12), and then, (13) can be obtained.

$$
D \tan \alpha+L-m g+m \frac{(M \cdot a)^{2}}{\left(R_{e}+h\right)}=0 .
$$

Then, a transcendental equation which is only related to $\alpha$ can be obtained by substituting (14) into (13). After the value of $\alpha$ is obtained, $s$ can be also calculated by (12).

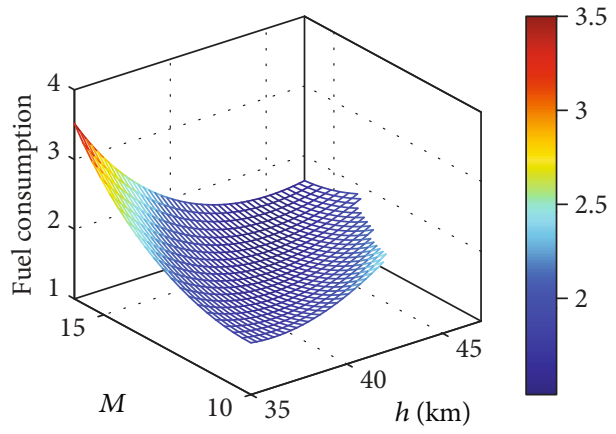

FIGURE 3: The distribution of fuel consumption averaged by range at different flight altitudes and Mach numbers.

$$
\begin{aligned}
& L=C_{L} \cdot \frac{1}{2} \rho(M \cdot a)^{2} \cdot S \\
& D=C_{D} \cdot \frac{1}{2} \rho(M \cdot a)^{2} \cdot S .
\end{aligned}
$$

Owning to that $\gamma$ is 0 while $h$ and $M$ remain unchanged. The fuel consumption averaged by flight range can be simplified as in (15) and [6]. Thus, all parameters in the trajectory of steady-state cruise can be solved.

$$
J=\frac{T}{g I_{\mathrm{sp}} M \cdot a}\left(1+\frac{h}{R_{e}}\right) .
$$




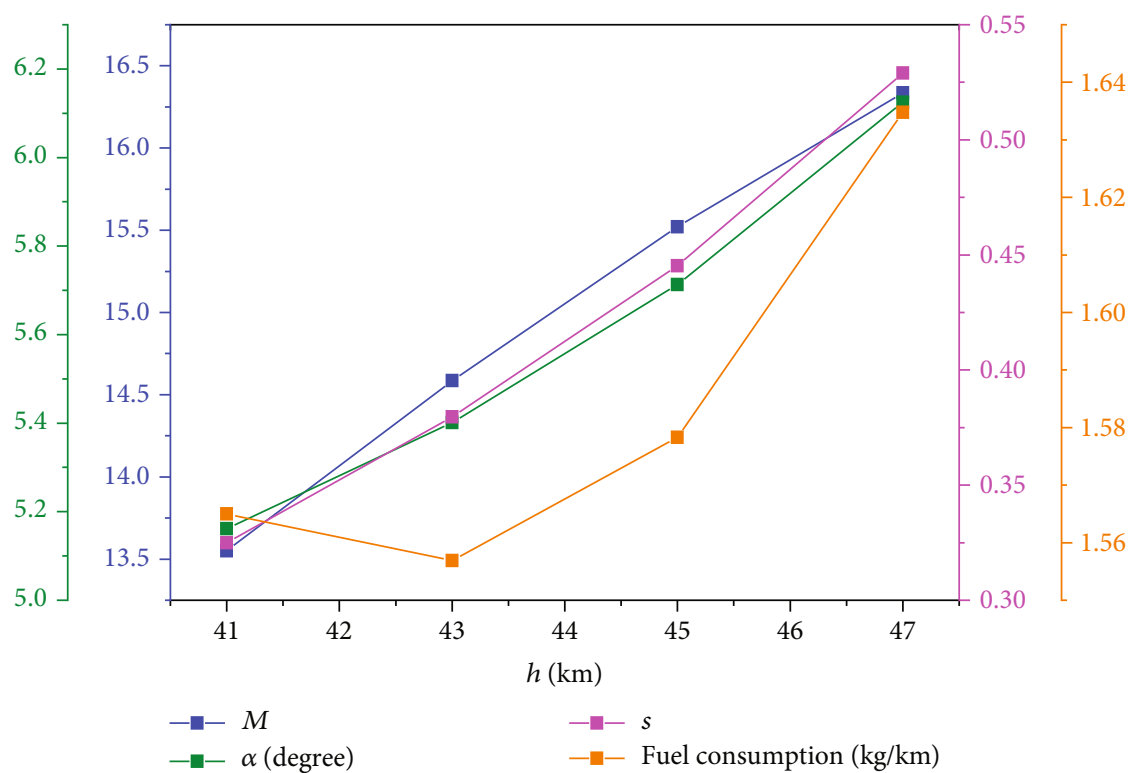

FIGURE 4: The local optimal parameters of steady-state cruise at different flight altitudes.

2.3. Optimization Problem of Periodic Cruise. In a periodic cruise whose period is donated by $t_{c}$, the terminal constraints are illustrated in (16).

$$
\left\{\begin{array}{l}
h(0)=h\left(t_{c}\right), \\
v(0)=v\left(t_{c}\right), \\
\gamma(0)=\gamma\left(t_{c}\right) .
\end{array}\right.
$$

The objective of optimization is to minimize the fuel consumption averaged by flight range in a cruise period, which is

$$
J=\frac{\int_{0}^{t_{c}}|\dot{m}| d t}{\int_{0}^{t_{c}} \dot{r} d t}=\frac{m(0)-m\left(t_{c}\right)}{r_{c}} .
$$

The limits of $\alpha$ and $s$ are determined according to the original data benchmark [15], as shown in Table 1.

Therefore, the optimization problem of periodic cruise trajectory can be formulated as (18).

$$
\begin{aligned}
& \text { Minimize } J=\frac{m\left(t_{c}\right)-m(0)}{r_{c}}, \\
& \text { subject to }\left\{\begin{array}{l}
\alpha(t) \in[5,20], \\
s(t) \in[0,1], \\
h(0)=h\left(t_{c}\right), \\
v(0)=v\left(t_{c}\right), \\
\gamma(0)=\gamma\left(t_{c}\right) .
\end{array}\right.
\end{aligned}
$$

In [31], based on a simplified dynamic model, the value of throttle in periodic cruise was solved by the minimum principle. It proved that the value of throttle was only 0 or 1 , and the engine only started once in a cycle. Gao et al. [15] regarded the highest point of periodic cruise trajectory as the starting point and assumed that the curve which depicted the change of altitude was approximately a cosine curve with the increase of range. The cosine curve was introduced into the optimal control problem as a path constraint, and the parameters which determined the shape of cosine curve were regarded as the augmented state. The optimized results were similar to that in [31]: the throttle switched between 0 and 1. Based on the results of above references, the flight plan of periodic cruise selected in this paper is as shown in Figure 1: the engine switches on when $\gamma$ is equal to 0 , and it lasts for 60 seconds; then, the aircraft begins to glide. The throttle is 1 when starting, and the rest is 0 .

2.4. Two-Level Optimization Method for Periodic Cruise. As shown in Figure 1, during the period, the engine switches on and off periodically; thus, the whole trajectory in a period can be divided into two phases. One is the acceleration phase when engine switches on and aircraft speeds up, and the other is the glide phase when engine switches off and aircraft glides without propulsion. Due to a relatively small proportion of acceleration phase [31], the angle of attack in this phase is regarded unchanged, and its value is regarded as a variable which needs to be optimized. Therefore, when studying the acceleration phase, it is a kind of parameter optimization problem. In this paper, the optimization about the angle of attack in the acceleration phase is carried out in the first level of the two-level method by the Downhill Simplex Method (DSM). The DSM is a geometrically intuitive algorithm. In two dimensions, the simplex is a triangle and it is a tetrahedron in three dimensions. As the algorithm proceeds, the simplex makes way downward toward the location of the minimum through series of steps.

The glide phase accounts for a large proportion in the whole flight period, and the angle of attack in this phase cannot be regarded as a constant. Therefore, it is a dynamic optimal control problem whose variables change with the increase of time. In the glide phase, there is no propulsion 


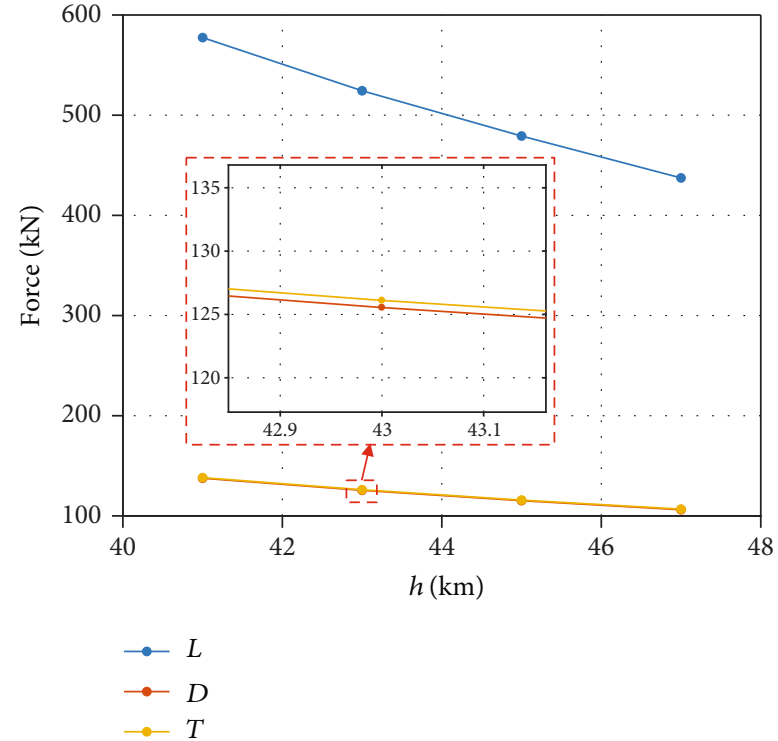

FIGURE 5: The value of lift, drag, and thrust of steady-state cruise at different flight altitudes.

and the throttle is 0 ; thus, the angle of attack is the only one control variable. In recent years, direct methods, especially Pseudo-Spectral Methods (PSMs), have become increasingly mature with the development of computers and optimization algorithm. A number of improvements for PSM and combinations with other algorithms were proposed [32]. The GPOPS (Gauss Pseudo-spectral Optimization Software), which is based on PSM and some effective nonlinear programming methods such as sequential quadratic programming (SQP), has been successfully applied in trajectory optimization problems. To optimize the angle of attack in the glide phase quickly and accurately, the GPOPS is employed as the second-level optimization, whose target is the longest flight range in the glide phase and the terminal constraint is (16).

Therefore, due to the division of the whole trajectory, the whole optimization is divided into a parameter optimization problem and an optimal control problem, which are solved in different levels, respectively. Thus, the two levels of the optimization method are mainly divided by different phases of trajectory. Aiming at the minimal fuel consumption averaged by flight range, the process of two-level optimization is shown in Figure 2 and the details are as follows:

Step 1. Set the initial value of angle of attack in the acceleration phase, and calculate the trajectory by the forth order Runge-Kutta (RK-4) method.

Step 2. Take the terminal point of acceleration phase as the starting point, and optimize the angle of attack in glide phase by the GPOPS, whose target is the longest flight range.

Step 3. Calculate the fuel consumption averaged by range in the whole period, and the result is fed back to the Downhill Simplex Method.

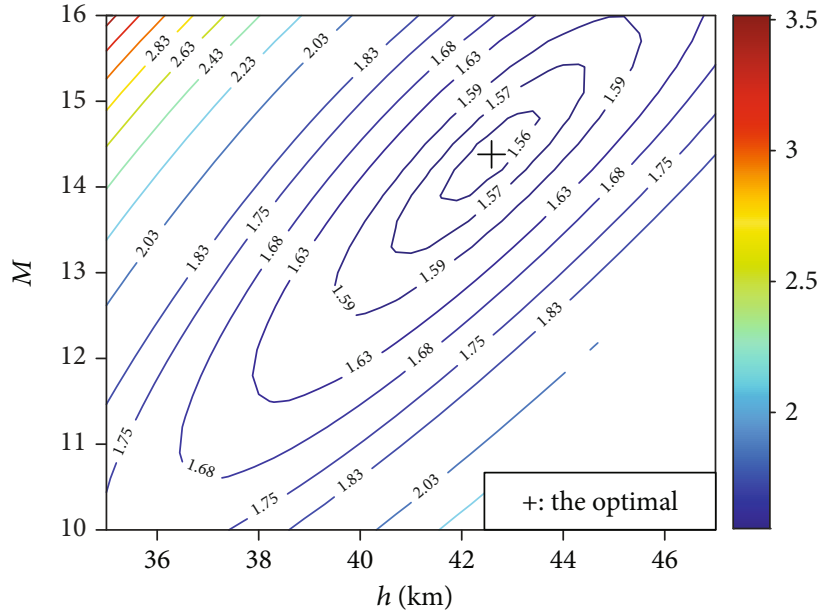

FIgURE 6: The contour of fuel consumption at different flight altitudes and Mach numbers.

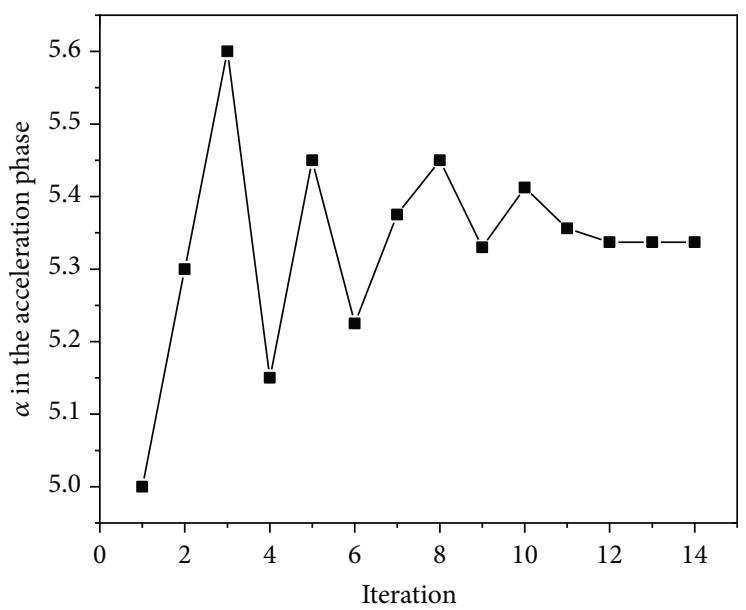

FIGURE 7: The change of angle of attack in the acceleration phase during the optimization process.

Step 4. Adjust the angle of attack in the acceleration phase by Downhill Simplex Method, and calculate the trajectory.

Step 5. Repeat Step 2 to Step 4 until the algorithm converges.

\section{Results and Analysis}

3.1. Result of Steady-State Cruise. In steady-state cruise, the distribution of fuel consumption averaged by range at different flight altitudes and Mach numbers is displayed in Figure 3. It can be seen that there is a minimal fuel consumption, whose value is $2 \mathrm{~kg} / \mathrm{km}$ lower than the maximum. Thus, the optimization of trajectory is significant to reduce fuel consumption.

At a constant flight altitude, with the change of Mach number, there is an optimal flight state whose fuel consumption is the minimum, and it is named the local optimum, while the state with the minimal fuel consumption at all flight altitudes is named the global optimum. Some parameters of local optimums at different flight altitudes are displayed in 


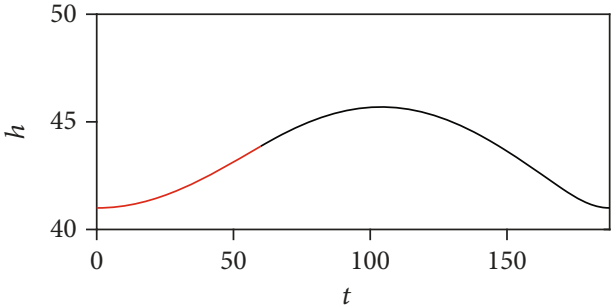

(a)

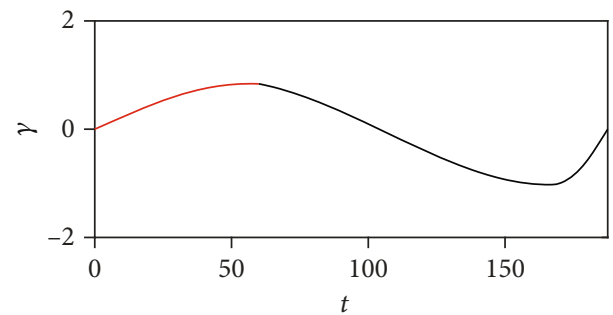

(c)

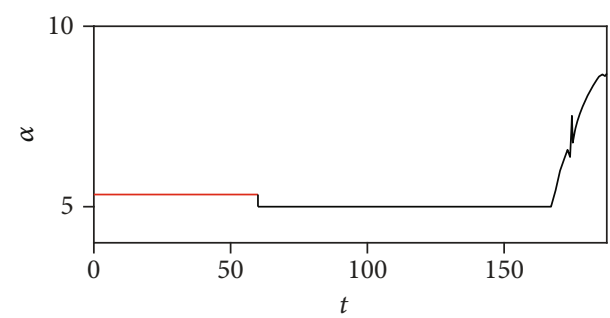

(e)

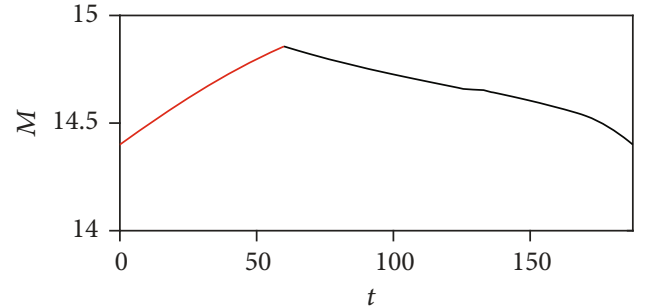

(b)

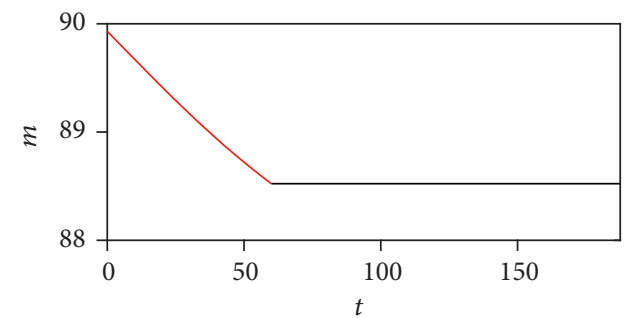

(d)

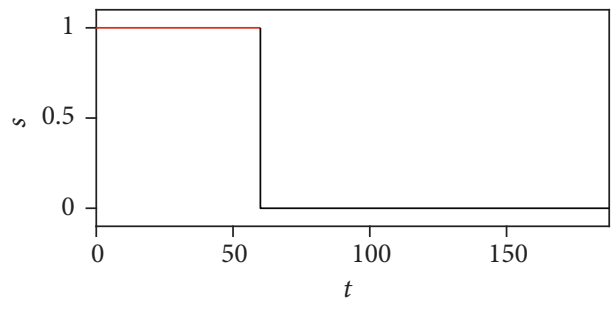

(f)

FIgURE 8: The flight parameters in the whole periodic cruise at $41 \mathrm{~km}$ : (a) flight altitude (km); (b) Mach number; (c) fight-path angle (degree); (d) mass (ton); (e) angle of attack (degree); (f) throttle.

Figure 4. With the enhancement of flight altitude, the local optimal Mach number and angle of attack as well as throttle increase, while the fuel consumption averaged by range decreases firstly and then increases. Thus, it can be concluded that the global optimal flight altitude of steady-state cruise is around $43 \mathrm{~km}$.

Figure 5 displays the change of lift, drag, and thrust with the increase of flight altitude. It can be seen that all forces decease if flight altitude is higher; thus, the increase of flight altitude tends to reduce the value of drag, which is also beneficial to reduce fuel consumption. However, from Figure 4, a higher flight altitude is accompanied with a higher flight Mach number, which will cause the decrease of impulse according to (4) and thus the efficiency of engine reduces. Therefore, there is an intervening flight altitude between the two aspects to obtain a relatively lower drag and a relatively higher impulse, and that is the point where the global optimum locates. Overall, the global optimal parameters of steady-state cruise trajectory are a result of the combination of the two aspects; thus, minimal fuel is consumed.

By the method of traversal, the flight altitude of the global optimum in steady-state cruise is at $42.6 \mathrm{~km}$ and the Mach number is 14.4 . The fuel consumption averaged by range is $1.556 \mathrm{~kg} / \mathrm{km}$. Figure 6 shows the contour of fuel consumption at different flight altitudes and Mach numbers. It can be seen that if one of the flight altitudes or Mach numbers is away from the global optimal point, more fuel will be consumed, and the total flight range will decrease. However, a hypersonic vehicle usually cannot always work at a state around the optimal point because of the constraints of structure and the requirements of mission. Therefore, it is significant to explore periodic cruise whose trajectory is more flexible with the fuel consumption reduced.

3.2. Optimization Results of Periodic Cruise. Based on the two-level optimization method, the starting point is set in the flight altitude at $41 \mathrm{~km}$ and Mach number at 14.4 . The initial value of optimization variable in the first level is set as $5^{\circ}$; then, the whole two optimization levels are implemented. Figure 7 shows the change of angle of attack in the acceleration phase during the optimization process, which is convergent at $5.337^{\circ}$ after 12 iterations of optimization. The complete trajectory and flight parameters in a whole period are displayed in Figure 8, where the trajectory of acceleration phase is depicted by red lines while that of glide phase is described by black lines. The fuel consumption averaged by range in the whole period is $1.514 \mathrm{~kg} / \mathrm{km}$, whose fuel-saving efficiency is $5.1 \%$ compared with that of steady-state cruise at $1.596 \mathrm{~kg} / \mathrm{km}$ at the same flight altitude and Mach number. Therefore, periodic cruise has more advantages in fuel saving, and the two-level optimization method is validated as well.

In last section, it is found that the flight altitude of optimal steady-state cruise point with the minimal fuel 


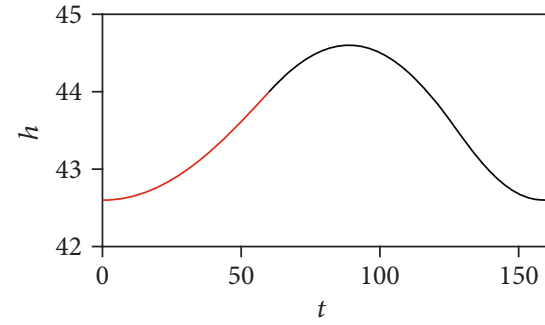

(a)

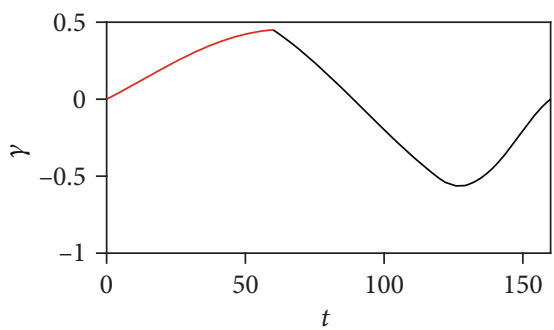

(c)

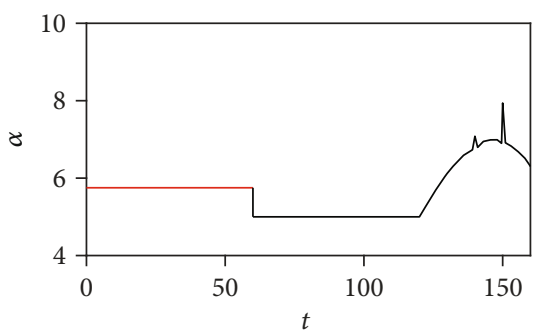

(e)

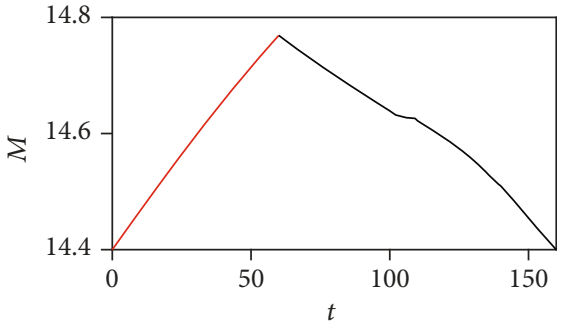

(b)

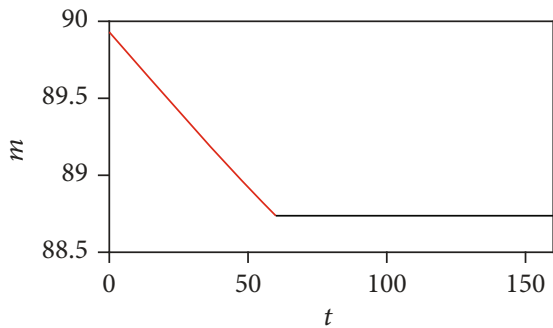

(d)

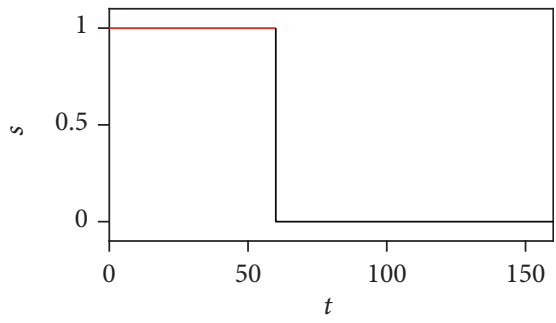

(f)

Figure 9: The flight parameters in the whole periodic cruise at $42.6 \mathrm{~km}$ : (a) flight altitude (km); (b) Mach number; (c) fight-path angle (degree); (d) mass (ton); (e) angle of attack (degree); (f) throttle.
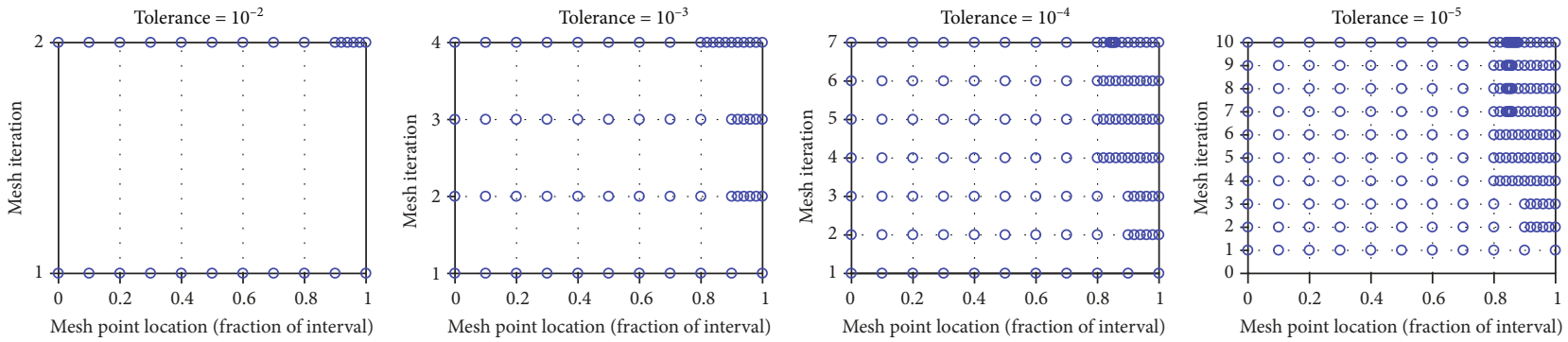

FIGURE 10: Mesh grid distribution with different accuracy tolerances.

consumption is at $42.6 \mathrm{~km}$ and the Mach number is 14.4 . To further validate the two-level optimization method and explore the performance of periodic cruise, optimization of periodic cruise is carried out at the optimal point of steadystate cruise. The initial value of angle of attack in the acceleration phase is set as $5^{\circ}$ as well. Figure 9 shows the optimized trajectory and flight parameters in the whole periodic cruise. The optimal value of angle of attack in the acceleration phase is $5.751^{\circ}$, and the fuel consumption averaged by range in the whole period is $1.511 \mathrm{~kg} / \mathrm{km}$, whose fuel-saving efficiency is $2.92 \%$ compared with that of steady-state cruise at 1.556 $\mathrm{kg} / \mathrm{km}$. A result with less fuel consumption can be obtained by the two-level optimization even at the optimal steady- state cruise point; thus, the effectiveness of optimization method proposed is proved further.

3.3. Analysis of Parameters in Method. To detail the influence of the GPOPS on the whole method, the distribution of mesh points with different tolerance is displayed in Figure 10. It can be seen that more mesh points are needed to achieve a higher accuracy. It is worth mention that the angle of attack in the acceleration phase changes in the optimization process, which generates different trajectories of the acceleration phase. Thus, the optimization of the trajectory in glide phase by the GPOPS is implemented in different starting points during the optimization process, while generally the GPOPS 


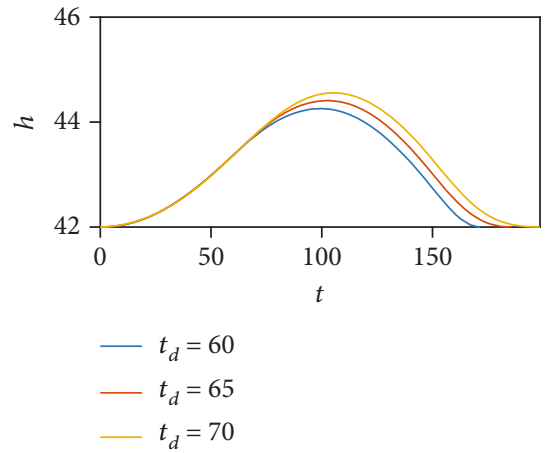

(a)

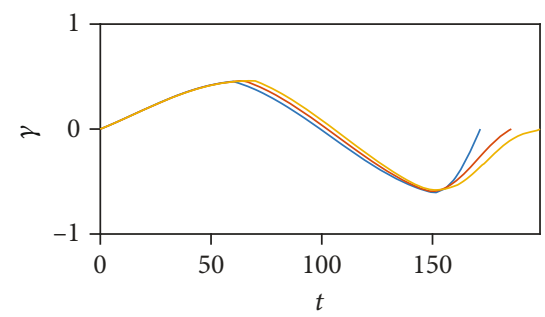

$$
\begin{aligned}
-t_{d} & =60 \\
-t_{d} & =65 \\
-t_{d} & =70
\end{aligned}
$$

(c)

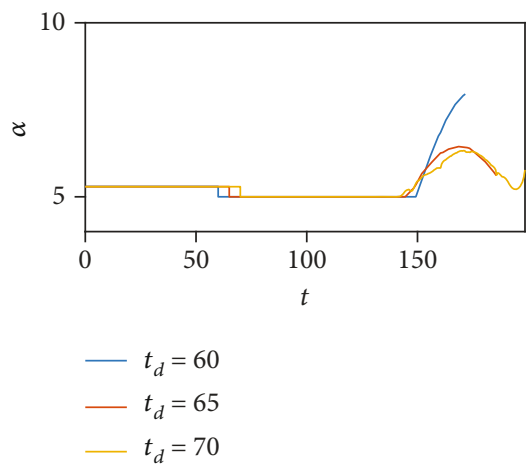

(e)

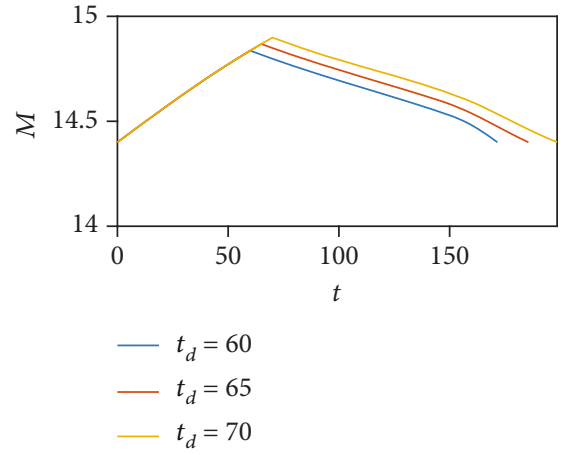

(b)

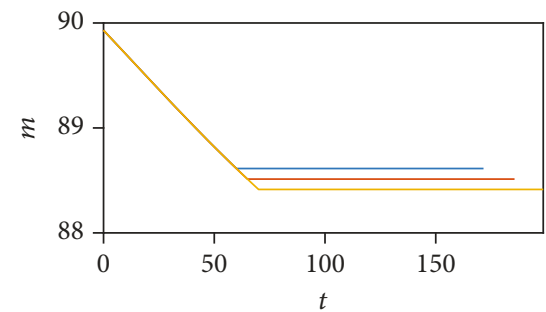

$$
\begin{aligned}
-t_{d} & =60 \\
-t_{d} & =65 \\
-t_{d} & =70
\end{aligned}
$$

(d)

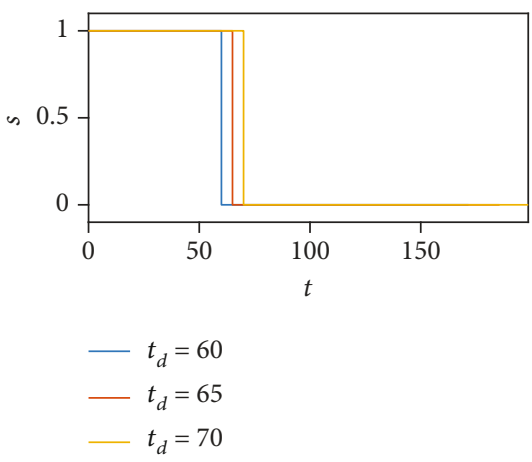

(f)

Figure 11: The flight parameters in the whole periodic cruise under different $t_{d}$ : (a) flight altitude (km); (b) Mach number; (c) fight-path angle (degree); (d) mass (ton); (e) angle of attack (degree); (f) throttle.

needs a lot of time to obtain a robust result with a small tolerance at most starting points. Thus, the optimization would cost more time if smaller tolerance is required, and the tolerance should be selected to balance computational accuracy and speed.

In addition, the length of duration when engine works, denoted by $t_{d}$, is set at $60 \mathrm{~s}$ in previous study. To detail the effect of $t_{d}$ on the method, a parameter variation of $t_{d}$ is carried out. When that value of $t_{d}$ is $60 \mathrm{~s}, 65 \mathrm{~s}$, and $70 \mathrm{~s}$, the optimal angle of attack in the acceleration phase is obtained by the proposed method and the value is $5.295^{\circ}, 5.291^{\circ}$, and $5.288^{\circ}$, respectively, which decrease slightly. And the reason should be that a high velocity can be guaranteed at the end of acceleration phase if $t_{d}$ is large, while a small angle of attack is beneficial to enhance the impulse and reduce the drag. As shown in Figure 11, different optimal trajectories are obtained by the proposed method, which confirm the applicability and feasibility.

3.4. Analysis of Periodic Cruise Trajectory. When the flight altitude of starting point is at $41 \mathrm{~km}$ and the Mach number is 14.4, a fuel-saving efficiency more than $5 \%$ is achieved by periodic cruise. Take this as an example to explore the mechanism of fuel saving in periodic cruise.

Figure 12 shows the change of drag during periodic cruise and steady-state cruise, respectively. Combined with Figure 8 , it can be seen that the aircraft accelerates and ascents with the propulsion provided by engine. Then, the engine switches off, and the aircraft begins to glide without power. At this time, the flight altitude is relatively high and 


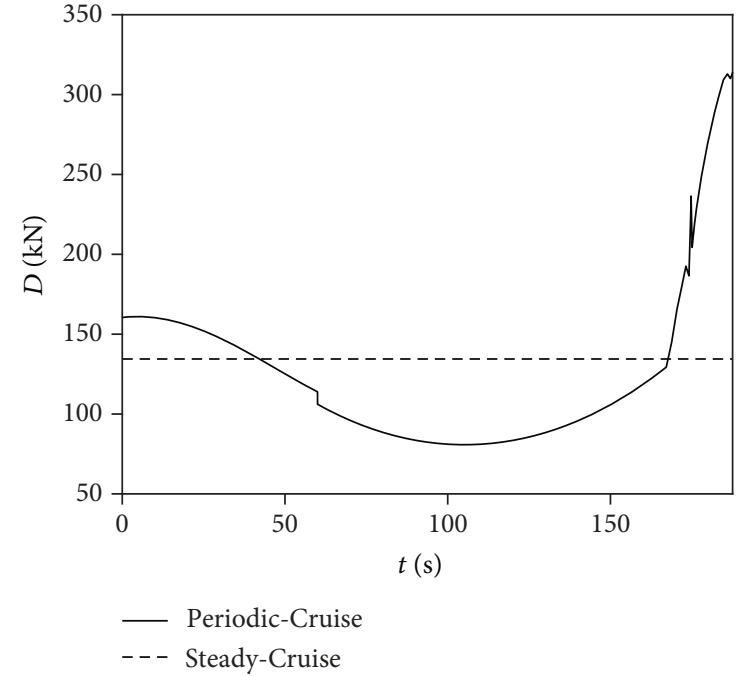

FIGURE 12: The change of drag in periodic cruise and steady-state cruise.

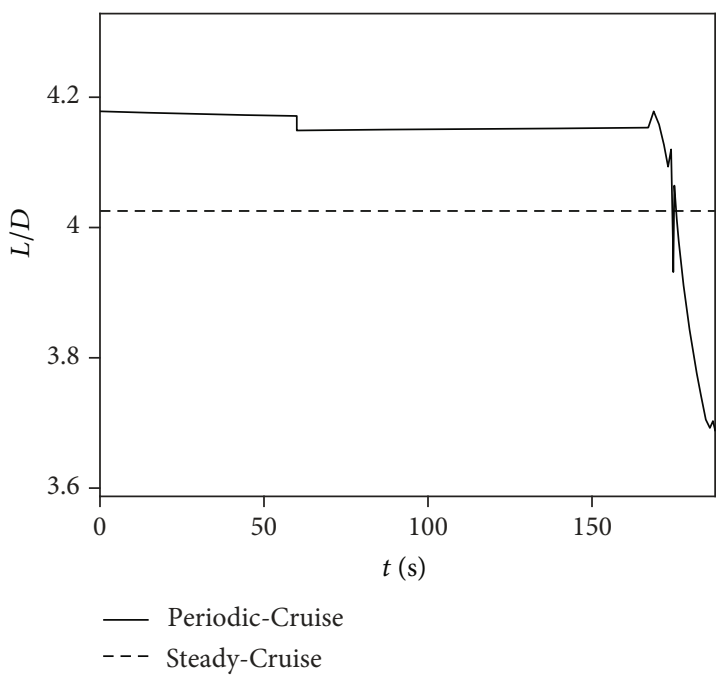

Figure 13: The change of lift-drag ratio in periodic cruise and steady-state cruise.

the atmospheric density is low, which is suitable for glide because of the small drag as shown in Figure 12. When the flight altitude is relatively low, the engine switches on again and the acceleration phase starts. At this time, the atmospheric density is large, which is beneficial for combustion in engine, and larger impulse is achieved according to (4). Therefore, the whole trajectory of periodic cruise shuttles between an atmosphere layer with relatively high density and an atmosphere layer with relatively low density, and it is similar to the ducks and drakes. The trajectory of periodic cruise makes full use of the change of atmospheric density to reduce drag in the glide phase and enhance impulse in the acceleration phase; thus, less fuel is consumed compared with that of steady-state cruise.

According to the Breguet Range Equation [33], a large lift-drag ratio is beneficial for a longer flight range. The change of lift-drag ratio in periodic cruise and steady-state cruise is displayed in Figure 13. It can be seen that the value of lift-drag ratio in periodic cruise is mostly between 4.1 and 4.2, while that of steady-state cruise is around 4.0, which is also a reason why fuel saving is achieved in periodic cruise.

Therefore, the trajectory of periodic cruise utilizes the change of atmospheric density to reduce drag and enhance impulse and adopts flight states with higher lift-drag ratio to enhance flight range. Therefore, less fuel is consumed compared with that of steady-state cruise.

\section{Conclusions}

Based on the characteristic of periodic cruise trajectory that the whole trajectory can be divided into an acceleration phase and a glide phase, a two-level optimization method which combines the Downhill Simplex Method with the PseudoSpectral Method is proposed in this paper; then, the trajectory of periodic cruise for hypersonic vehicle is optimized and analyzed. Conclusions can be drawn as follows:

(1) The proposed optimization method can deal with the design problem of periodic cruise trajectory with feasibility confirmed

(2) The fuel consumption averaged by range in periodic cruise trajectory is less than that of steady-state cruise trajectory, which means periodic cruise can save a certain amount of fuel and make range longer

(3) The trajectory of periodic cruise makes more use of atmospheric density and lift-drag ratio of aircraft, and therefore, fuel saving is achieved

In future work, the length of duration when engine works can be also regarded as an optimization variable, while more improvements are needed to make sure credible results can be obtained by PSM. To enhance the accuracy of results and make the method more robust, new method, such as initial guess generator technique, can be introduced.

\section{Data Availability}

The image data used to support the findings of this study are included within the article.

\section{Conflicts of Interest}

The authors declare that there is no conflict of interest regarding the publication of this paper.

\section{Acknowledgments}

This research was funded by the Postgraduate Scientific Research Innovation Project of Hunan Province (Grant number: CX20200084). The authors want to express their thanks for the support from Tao Tang.

\section{References}

[1] Z. Chen and Y. Zhao, "Aerothermoelastic analysis of a hypersonic vehicle based on thermal modal reconstruction," 
International Journal of Aerospace Engineering, vol. 2019, 13 pages, 2019.

[2] J. Zhao and R. Zhou, "Reentry trajectory optimization for hypersonic vehicle satisfying complex constraints," Chinese Journal of Aeronautics, vol. 26, no. 6, pp. 1544-1553, 2013.

[3] X. Zhang, J. Huang, G. Wang, and L. Li, "Hypersonic target tracking with high dynamic biases," IEEE Transactions on Aerospace \& Electronic Systems, vol. 55, no. 1, pp. 506-510, 2019.

[4] Y. Fan, P. Yan, F. Wang, and H. Xu, "Discrete sliding mode control for hypersonic cruise missile," International Journal of Aerospace Engineering, vol. 2016, 9 pages, 2016.

[5] G. N. Kumar, M. Ikram, and A. K. Sarkar, "Hypersonic flight vehicle trajectory optimization using pattern search algorithm," Optimization and Engineering, vol. 19, no. 1, pp. 125-161, 2018.

[6] X. Liu, Y. Wang, L. Liu, and J. Huang, "Optimization on steady-state cruise for a hypersonic vehicle," in Proceedings of the 33rd Chinese Control Conference, pp. 8935-8940, Nanjing, China, 2014.

[7] B. Pan and P. Lu, "Improvements to optimal launch ascent guidance," in AIAA Guidence, Navigation, and Control Conference, vol. 8174, pp. 2-5, Toronto, Ontario, Canada, 2012.

[8] G. Cai, G. Duan, C. Hu, and B. Zhou, "Tracking control for airbreathing hypersonic cruise vehicle based on tangent linearization approach," Journal of Systems Engineering and Electronics, vol. 21, no. 3, pp. 469-475, 2010.

[9] Z. Huang, Y. Zhang, and Y. Liu, "Research on state estimation of hypersonic glide vehicle," Journal of Physics: Conference Series, vol. 1060, no. 1, article 012088, 2018.

[10] M. Xu, L. Liu, G. Tang, and K. Chen, "Study on guidance approaches of hypersonic glide-cruise vehicle during cruise and attack phases," in Proceedings of 3rd International Symposium on Systems and Control in Aeronautics and Astronautics, pp. 1491-1495, Harbin, China, 2010.

[11] P. F. Gath and A. J. Calise, "Optimization of launch vehicle ascent trajectories with path constraints and coast arcs," Journal of Guidance, Control, and Dynamics, vol. 24, no. 2, pp. 296-304, 2001.

[12] D. E. Koelle, "On the optimum cruise speed of a hypersonic aircraft," IEEE Aerospace and Electronic Systems Magazine, vol. 4, no. 5, pp. 13-16, 1989.

[13] K. Wang, B. Zhang, and Y. Hou, "Multiobjective optimization of steady-state cruise trajectory for a hypersonic vehicle," in 2017 3rd IEEE International Conference on Control Science and Systems Engineering (ICCSSE), pp. 130-135, Beijing, 2017.

[14] J. R. Irons, R. L. Schultz, and N. R. Zagalsky, "Energy state approximation and minimum-fuel fixed-range trajectories," Journal of Aircraft, vol. 8, no. 6, pp. 488-490, 1971.

[15] H. Gao, Z. Chen, M. Sun, Z. Wang, and Z. Chen, "General periodic cruise guidance optimization for hypersonic vehicles," Applied Sciences, vol. 10, no. 8, p. 2898, 2020.

[16] P. Menon, G. Sweriduk, and A. Bowers, "A study of nearoptimal endurance-maximizing periodic cruise trajectories, AIAA 2005-6153," in AIAA Guidance, Navigation, and Control Conference and Exhibit, San Francisco, California, 2005.

[17] C. F. Ollivier-Gooch and M. Azab, "High-order aerodynamic optimization using new hybrid sequential quadratic programing-particle swarm intelligence technique," in 50th AIAA Aerospace Sciences Meeting including the New Horizons Forum and Aerospace Exposition, 2012.
[18] D. Spiller, L. Ansalone, and F. Curti, "Particle swarm optimization for time-optimal spacecraft reorientation with keep-out cones," Journal of Guidance, Control, and Dynamics, vol. 39, pp. 312-325, 2016.

[19] B. A. Conway, "A survey of methods available for the numerical optimization of continuous dynamic systems," Journal of Optimization Theory and Applications, vol. 152, no. 2, pp. 271-306, 2012.

[20] J. T. Betts, "Survey of numerical methods for trajectory optimization," Journal of guidance, control, and dynamics, vol. 21, pp. 193-207, 1998.

[21] W. Wang, Z. Hou, S. Shan, and L. Chen, "Optimal periodic control of hypersonic cruise vehicle: trajectory features," IEEE Access, vol. 7, pp. 3406-3421, 2019.

[22] P. H. Carter, D. J. Pines, and L. V. Rudd, “Approximate performance of periodic hypersonic cruise trajectories for global reach," IBM Journal of Research and Development, vol. 35, no. 5, pp. 703-714, 2000.

[23] G. Huang, Y. Lu, and Y. Nan, "A survey of numerical algorithms for trajectory optimization of flight vehicles," Science China Technological Sciences, vol. 55, no. 9, pp. 2538-2560, 2012.

[24] K. Subbarao and B. M. Shippey, "Hybrid genetic algorithm collocation method for trajectory optimization," Journal of Guidance, Control, and Dynamics, vol. 32, no. 4, pp. 1396-1403, 2009.

[25] B. N. Kang, D. B. Spencer, S. Tang, and D. Jordan, “Optimal periodic cruise trajectories via a two-level optimization method," Journal of Spacecraft and Rockets, vol. 47, no. 4, pp. 597-613, 2015.

[26] R. Chai, A. Savvaris, A. Tsourdos, S. Chai, and Y. Xia, "Trajectory optimization of space maneuver vehicle using a hybrid optimal control solver," IEEE Transactions on Cybernetics, vol. 49, pp. 467-480, 2017.

[27] R. Chai, A. Tsourdos, A. Savvaris, Y. Xia, and S. Chai, "Realtime reentry trajectory planning of hypersonic vehicles: a two-step strategy incorporating fuzzy multi-objective transcription and deep neural network," IEEE Transactions on Industrial Electronics, vol. 67, pp. 6904-6915, 2019.

[28] C. Chuang and H. Morimoto, "Optimal periodic cruise for a hypersonic vehicle with constraints," in Guidance, Navigation, and Control Conference, San Diego, CA, U.S.A., 1996.

[29] G. M. Ware and C. I. Cruz, "Aerodynamic characteristics of the HL-20," Journal of spacecraft and rockets, vol. 30, no. 5, pp. 529-536, 1993.

[30] C. H. Chuang and H. Morimoto, "Periodic optimal cruise for a hypersonic vehicle with constraints," Journal of spacecraft and rockets, vol. 34, no. 2, pp. 165-171, 1997.

[31] R. Li and Y. Shi, "The fuel optimal control problem of a hypersonic aircraft with periodic cruising mode," Mathematical and Computer Modelling, vol. 55, no. 11-12, pp. 2141-2150, 2012.

[32] D. Christopher, H. William, and R. Anil, “An improved adaptive hp algorithm using pseudospectral methods for optimal control," in AIAA/AAS Astrodynamics Specialist Conference, Toronto, Ontario, Canada, 2010.

[33] L. Bréguet, "Calcul du poids de combustible consummé par un avionen vol ascendant," Comptes Rendus del'Academie des Sciences, vol. 177, pp. 870-872, 1923. 